\title{
EXACT ASYMPTOTIC BEHAVIOR OF THE PEKAR-TOMASEVICH FUNCTIONAL
}

\author{
RAFAEL D. BENGURIA ${ }^{1}$ AND GONZALO A. BLEY ${ }^{2}$
}

\begin{abstract}
An explicit asymptotic expression for the ground-state energy of the PekarTomasevich functional for the $\mathrm{N}$-polaron is found, when the repulsion parameter $U$ of the electrons satisfies the inequality $0 \leq U \leq 2 \alpha$, where $\alpha$ is the coupling constant of the polaron. If $\mathcal{E}_{U}^{N}$ denotes this ground-state energy for the case of $N$ electrons and repulsion parameter $U$, we prove that $\mathcal{E}_{U}^{N} / N^{3} \rightarrow-c_{p}(U-2 \alpha)^{2} / 4$ as $N \rightarrow \infty$, where, $c_{p}=0.10851 \ldots$ Moreover, we show that $\mathcal{E}_{0}^{N}=-c_{p} \alpha^{2} N^{3}$, for all $N$.
\end{abstract}

\section{INTRODUCTION}

The Pekar-Tomasevich functional arises as an upper bound for the ground-state energy of the Hamiltonian of the N-polaron. We shall begin by explaining what is understood by a polaron: it was first considered by Fröhlich [6, 7] as a model of one non-relativistic electron interacting with the longitudinal, optical phonons in the periodic lattice of atoms of a polar crystal (e.g. $\mathrm{NaCl}$ ). More precisely, the polaron is the ensemble electron plus the polarization of the lattice, created by this electron. It was later called the "large" polaron, since in the formulation of the model, it was assumed that the distortion of the lattice, due to the electron, comprised several lattice constants, and so the medium was treated as a continuum. For a self-contained introduction to polarons, the reader may consult [18. Also, a very complete review of what was known about it, until 1969, is in [1]. The N-polaron is the extension of the above situation to $\mathrm{N}$ electrons, interacting not only with the field of phonons, but also with each other through their mutual Coulomb repulsion.

The Hamiltonian of the polaron is given by,

$$
H^{1}=p^{2}-\frac{1}{\pi} \sqrt{\frac{\alpha}{2}} \int\left[a(k) e^{i k x}+a(k)^{\dagger} e^{-i k x}\right] \frac{d k}{|k|}+\int a(k)^{\dagger} a(k) d k .
$$

As usual, whenever we omit the subscript in the integrals, integration in $\mathbb{R}^{3}$ is understood. Here, $x \in \mathbb{R}^{3}$ is the position of the electron, and $p$ is the corresponding momentum. Moreover, $a(k)$ denotes the lowering operator for the harmonic oscillator corresponding to the mode $k \in \mathbb{R}^{3}$ of the field, and this operator and its adjoint satisfy the canonical commutation relation $\left[a(k), a\left(k^{\prime}\right)^{\dagger}\right]=\delta\left(k-k^{\prime}\right)$, where $\delta$ is the Dirac delta. The first term corresponds to the kinetic energy of the electron ( $p$ is given by $-i \nabla$ ), the second one to the interaction of the electron with the phonon field, and the last term is the energy of this field. We use units such that $\hbar=2 m=\omega=1$, where $m$ is the band mass of the electron, and $\omega$ is the frequency of the phonons. We remark the fact that the dispersion relation is indeed $\omega=1$, that is, there is no dependence of the frequency of the phonons upon the mode of the field; this is part of the assumptions made for the derivation of this Hamiltonian by Fröhlich. The operator $H^{1}$ acts on the Hilbert space $L^{2}\left(\mathbb{R}^{3}\right) \otimes \mathcal{F}$, where $\mathcal{F}$ is the Fock space associated to the phonons. 
$\alpha$ is a positive, adimensional constant, known as the coupling constant for the polaron, and there is an explicit expression for it; restoring units, it is given by,

$$
\alpha=\frac{e^{2}}{2 \hbar \omega}\left(\frac{2 m \omega}{\hbar}\right)^{1 / 2}\left(\frac{1}{\epsilon_{\infty}}-\frac{1}{\epsilon_{0}}\right)
$$

where $\omega$ is as before, the frequency of the phonons, $e$ is the charge of the electron, and $\epsilon_{0}$ and $\epsilon_{\infty}$ are known as the static and high-frequency dielectric constants of the material, respectively.

The N-polaron Hamiltonian is simply a generalization of the previous operator, and is obtained by including the interelectronic repulsion:

$$
\begin{aligned}
H_{U}^{N}= & \sum_{i=1}^{N} p_{i}^{2}-\frac{1}{\pi} \sqrt{\frac{\alpha}{2}} \sum_{i=1}^{N} \int\left[a(k) e^{i k x_{i}}+a(k)^{\dagger} e^{-i k x_{i}}\right] \frac{d k}{|k|} \\
& +\int a(k)^{\dagger} a(k) d k+U \sum_{i<j} \frac{1}{\left|x_{i}-x_{j}\right|},
\end{aligned}
$$

where $x_{i}$ and $p_{i}$ are the position and the momentum of the $i$-th particle. $U$ is, in this units, an adimensional parameter, and it corresponds to a positive repulsion parameter. It is given by $e^{2} / \epsilon_{\infty}$, and it satisfies an important relation: note that,

$$
\alpha<\frac{e^{2}}{2 \hbar \omega}\left(\frac{2 m \omega}{\hbar}\right)^{1 / 2} \frac{1}{\epsilon_{\infty}}
$$

and so we must have $U>2 \alpha$. Nonetheless, the operator $H_{U}^{N}$ still makes sense when $0 \leq U \leq$ $2 \alpha$.

Given $N$, the number of electrons, the ground-state energy of $H_{U}^{N}$ ( $H_{U}^{1}$ being written as $H^{1}$ ), denoted by $E_{U}^{N}$, will be defined by,

$$
E_{U}^{N}=\inf \left\{\left(\psi, H_{U}^{N} \psi\right): \psi \in L^{2}\left(\mathbb{R}^{3 N}\right) \otimes \mathcal{F},\|\psi\|=1\right\} .
$$

As can be seen from the definition of $E_{U}^{N}$, we will not treat the electrons as fermions, spin will also be discarded. Moreover, $E_{U}^{1}$ will be denoted simply by $E^{1}$.

When $\alpha$ is sufficiently large, Pekar [20, 21] suggested that the ground state of (1) could be approximated by a product function $\phi \xi$, where $\phi$ involved only the coordinates of the electron, $\xi$ was a state of the phonon field, and both elements of the respective Hilbert spaces were normalized. It is clear that the expectation value of $H^{1}$ with such a function will overestimate the real ground-state energy. Pekar and Tomasevich [22] extended this idea to the bipolaron $(N=2)$. The case of arbitrary $N$ has been apparently considered for the first time by Frank, Lieb, Seiringer and Thomas [3, 4, 5]; they have only briefly sketched the derivation of the Pekar-Tomasevich functional for $N$ electrons, so for completeness we will give a more detailed calculation of this functional below. However, no part of the derivation will be mentioned in the rest of this article.

When a function of the form $\Psi=\psi \xi$, where this time $\psi \in H^{1}\left(\mathbb{R}^{3 N}\right)$, and $\|\psi\|=1$, is used as a trial function to estimate the ground-state energy of $(3),\left(\Psi, H_{U}^{N} \Psi\right)$ may be calculated as $\left\langle\xi\left|\left\langle\psi\left|H_{U}^{N}\right| \psi\right\rangle\right| \xi\right\rangle .\left\langle\psi\left|H_{U}^{N}\right| \psi\right\rangle$ can then be written, after completing the square, as,

$$
T+V+\int c(k)^{\dagger} c(k) d k-\frac{\alpha}{2 \pi^{2}} \sum_{i, j} \int \frac{b_{i}(k) \overline{b_{j}(k)}}{|k|^{2}} d k
$$


where $T$ is the kinetic energy of the electrons, $V$ is their repulsive energy, and $b_{i}(k)$ and $c(k)$ are defined as,

$$
\begin{gathered}
b_{i}(k)=\int e^{i k x_{i}}|\psi|^{2} d X, \\
c(k)=a(k)-\frac{1}{\pi} \sqrt{\frac{\alpha}{2}} \sum_{i=1}^{N} \frac{\overline{b_{i}(k)}}{|k|} .
\end{gathered}
$$

The last term of (6) is equal to,

$$
-\alpha \iint \frac{\rho(x) \rho(y)}{|x-y|} d x d y
$$

with $\rho$ being defined as,

$$
\rho(x)=\sum_{i=1}^{N} \int\left|\psi\left(x_{1}, \ldots, x, \ldots, x_{N}\right)\right|^{2} \widehat{d x}_{i},
$$

which is the single particle density, except that there is no multiplication by the charge of the electron. $\widehat{d x}_{i}$ denotes integration in every particle coordinate, except the $i$-th.

On the other hand,

$$
\int\left\langle\xi\left|c(k)^{\dagger} c(k)\right| \xi\right\rangle d k \geq 0
$$

and equality is attained, provided $\xi$ is a state such that, for each $k$, is given by the coherent state $|\beta(k)\rangle$ satisfying,

$$
a(k)|\beta(k)\rangle=\frac{1}{\pi} \sqrt{\frac{\alpha}{2}} \sum_{i=1}^{N} \frac{\overline{b_{i}(k)}}{|k|}|\beta(k)\rangle,
$$

(see, e.g., Lemma A.2 in [10, and (A.8) in [4]) and since the mode $k=0$ can be omitted in the integration, any state can be chosen for that vector, for instance $|0\rangle$ (the first eigenvector of the harmonic oscillator). Thus, the following functional of $\psi$ is found,

$$
\mathcal{P}_{U}^{N}(\psi)=\sum_{i=1}^{N} \int\left|\nabla_{i} \psi\right|^{2} d X+U \sum_{i<j} \int \frac{|\psi|^{2}}{\left|x_{i}-x_{j}\right|} d X-\alpha \iint \frac{\rho(x) \rho(y)}{|x-y|} d x d y,
$$

which will be called the Pekar-Tomasevich functional. Its ground-state energy, the infimum of the set of all numbers $\mathcal{P}_{U}^{N}(\psi)$, with $\psi$ in $H^{1}\left(\mathbb{R}^{3 N}\right)$ and $\|\psi\|=1$ will be denoted by $\mathcal{E}_{U}^{N}$. We will also write $\mathcal{P}_{0}^{1}$ as $\mathcal{P}$, and $\mathcal{E}_{0}^{1} \equiv \mathcal{E}$.

The case for $N=1$,

$$
\mathcal{P}(\phi)=\int|\nabla \phi|^{2} d x-\alpha \iint \frac{|\phi(x)|^{2}|\phi(y)|^{2}}{|x-y|} d x d y
$$

which we shall refer to as the Pekar functional, was studied by Lieb [12]. He found that there exists a unique normalized minimizer (up to translations and a phase). By scaling, that is, writing $\phi$ as $\lambda^{3 / 2} f(\lambda x)$, it is seen that its ground-state energy is $-c_{p} \alpha^{2}$, with $c_{p}$ being a constant. It has been found numerically that, to five decimals of precision, $c_{p}=0.10851$ [8].

This ground-state energy is asymptotically exact as $\alpha \rightarrow \infty$, in the sense that,

$$
\lim _{\alpha \rightarrow \infty} \frac{E^{1}(\alpha)}{\alpha^{2}}=-c_{p}
$$


a result that was proved by Donsker and Varadhan [2]; it was then rederived by Lieb and Thomas [16]. A similar result has been shown to hold by Miyao and Spohn for the bipolaron [19],

$$
\lim _{\alpha \rightarrow \infty} \frac{E_{U}^{2}(\alpha)}{\alpha^{2}}=2 c_{p}-\mathcal{E}_{U}^{2}(\alpha=1)
$$

When $U>2 \alpha$, Frank et al. [4] have proved that $\mathcal{E}_{U}^{N} \geq-C(U) N$, where $C(U)$ is a positive function that depends on $U$, but not on $N$. They have also shown a similar result for the Fröhlich Hamiltonian, thus settling stability of the second kind (boundedness from below of the energy divided by the number of particles) in this region; it also proves that $N$ is the correct asymptotic behavior, because a general result known as the subadditivity of the energy [9, 10, 15],

$$
E_{U}^{L} \leq E_{U}^{N}+E_{U}^{M}
$$

where $L=N+M$, holds. It is also valid for the ground-state energy of the Pekar-Tomasevich functional. These results not only show what the correct asymptotic behavior is, but also prove the existence of the thermodynamic limit,

$$
\lim _{N \rightarrow \infty} \frac{E_{U}^{N}}{N}
$$

which follows from the fact that $E_{U}^{N} / N$ is a bounded and monotone sequence of real numbers.

The case $0 \leq U \leq 2 \alpha$ for the Fröhlich Hamiltonian has been studied by Griesemer and Møller [10], at least when the electrons are treated as fermions. They have investigated $E_{U}^{N}$ and have obtained upper and lower bounds of the form $-A(U) N^{7 / 3}$, where $A$ is some function of $U$, thus establishing the correct asymptotic behavior when antisymmetric functions are used; however their bounds do not allow the obtention of an exact expression for $\lim _{N \rightarrow \infty} E_{U}^{N} / N^{7 / 3}$.

We have found that when $0 \leq U \leq 2 \alpha$, the ground-state energy of the Pekar-Tomasevich functional, divided by $N^{3}$, has a limit, given by,

$$
\lim _{N \rightarrow \infty} \frac{\mathcal{E}_{U}^{N}(\alpha)}{N^{3}}=-\frac{c_{p}}{4}(U-2 \alpha)^{2}
$$

which may be used as an approximation formula for large enough $N$. For $U=0$, we also prove the stronger result that $\mathcal{E}_{0}^{N}=-c_{p} \alpha^{2} N^{3}$, for all $N$. The situation $U=2 \alpha$ is still an open question, a discussion of this case is given below.

Though the result (19) is not directly applicable to the N-polaron model (due to the relationship between $U$ and $\alpha$ ), there may be other situations in which the Pekar-Tomasevich functional arises, and the condition $U>2 \alpha$ is no longer necessary. In fact, the Pekar functional appears also in another context, namely as the variational principle for the Choquard nonlinear equation [12, which arises as an approximation to the Hartree-Fock theory of a plasma.

\section{Main Results}

In this section we prove our main result concerning the exact asymptotic behavior of the Pekar-Tomasevich functional. We begin with the following theorem concerning the properties of $\mathcal{E}_{U}^{N}$.

Theorem 2.1 (Properties of $\mathcal{E}_{U}^{N}$ ).

(1) For all $N, \mathcal{E}_{0}^{N}=-c_{p} \alpha^{2} N^{3}$. 
(2) $\mathcal{E}_{U}^{N}$ is monotone non-decreasing as a function of $U, N$ being fixed, and thus $\mathcal{E}_{U}^{N}$ is finite for $U>0$.

(3) $\mathcal{E}_{U}^{N}$ is concave for $U>0, N$ being fixed, and thus it is a continuous function on $(0, \infty)$.

Proof. (i) Using the Hoffmann-Ostenhof bound [11,

$$
\sum_{i=1}^{N} \int\left|\nabla_{i} \psi\right|^{2} d x \geq \int|\nabla \sqrt{\rho}|^{2} d x
$$

one may then utilize directly the described properties of $(14)$ : in fact, writing $\phi \equiv \sqrt{\rho / N}$, and noting that $\|\phi\|_{2}=1$, we have that,

$$
\mathcal{P}_{0}^{N}(\psi) \geq N\left[\int|\nabla \phi|^{2} d x-\alpha N \iint \frac{\phi(x)^{2} \phi(y)^{2}}{|x-y|} d x d y\right]
$$

but this is precisely $N$ times the Pekar functional $(N=1)$ with parameter $\alpha N$, for which a minimizer exists, thus showing that $\mathcal{E}_{0}^{N} \geq-c_{p} \alpha^{2} N^{3}$. To obtain the corresponding upper bound, a product function can be used in (13),

$$
\prod_{i=1}^{N} \phi\left(x_{i}\right)
$$

and then by choosing $\phi$ as the minimizer of the Pekar functional with parameter $\alpha N$, the result is obtained.

(ii) This follows by noting that If $V>U, \mathcal{P}_{V}(\psi)>\mathcal{P}_{U}(\psi)$.

(iii) Since $\mathcal{P}_{U}^{N}(\psi)$, given by 13 is linear in $U$, the concavity of $\mathcal{E}_{U}^{N}$ follows at once from its definition as a minimization principle. Also, the continuity follows from the concavity.

Theorem 2.2. If $0<U<2 \alpha$,

$$
\lim _{N \rightarrow \infty} \frac{\mathcal{E}_{U}^{N}}{N^{3}}=-\frac{c_{p}}{4}(U-2 \alpha)^{2}
$$

Proof. Here, we proceed as in the proof of Proposition 1, Section 5, of [4]. As in the previous theorem, in order to get an upper bound for $\mathcal{E}_{U}^{N} / N^{3}$, consider as a trial function the product,

$$
\psi_{0} \equiv \prod_{i=1}^{N} \phi\left(x_{i}\right)
$$

where $\phi$ is, for now, an arbitrary function such that $\int|\phi(x)|^{2} d x=1$. It follows that $\mathcal{P}_{U}^{N}\left(\psi_{0}\right)$ is given by,

$$
N\left\{\int|\nabla \phi|^{2} d x-\left[N \alpha-\frac{U}{2}(N-1)\right] \iint \frac{|\phi(x)|^{2}|\phi(y)|^{2}}{|x-y|} d x d y\right\} .
$$

Since we are assuming $U<2 \alpha$, the expression in square brackets is positive for all $N$, and then this upper bound is optimized when $\phi$ is precisely the function that minimizes the Pekar functional when the parameter $\alpha$ is replaced by $[N \alpha-(U / 2)(N-1)]$. Thus, recalling that the ground-state energy of the Pekar functional with parameter $\alpha$ is given by $-c_{p} \alpha^{2}$, we obtain,

$$
\mathcal{E}_{U}^{(N)} \leq-\frac{c_{p}}{4}\left(U-2 \alpha-\frac{U}{N}\right)^{2} N^{3}
$$


which is an upper bound of the required form, except for a term, $-U / N$, that vanishes as $N \rightarrow \infty$.

For a lower bound, we will first use the Lieb-Oxford inequality [14],

$$
\sum_{i<j} \int \frac{|\psi|^{2}}{\left|x_{i}-x_{j}\right|} d X \geq \frac{1}{2} \iint \frac{\rho(x) \rho(y)}{|x-y|} d x d y-1.68 \int \rho^{4 / 3} d x
$$

together with the Hoffmann-Ostenhof inequality, and, as in the previous theorem, setting $\phi \equiv \sqrt{\rho / N}$, so as to have $\int \phi^{2} d x=1$, we obtain as a lower bound for $\mathcal{P}_{U}^{(N)}$,

$$
N\left[\int|\nabla \phi|^{2} d x-\frac{N}{2}(2 \alpha-U) \iint \frac{\phi(x)^{2} \phi(y)^{2}}{|x-y|} d x d y-1.68 U N^{1 / 3} \int \phi^{8 / 3} d x\right] .
$$

Next, let $\lambda \in(0,1)$, and write the previous expression as,

$$
\begin{aligned}
& N\left\{\lambda\left[\int|\nabla \phi|^{2} d x-\frac{N(2 \alpha-U)}{2 \lambda} \iint \frac{\phi(x)^{2} \phi(y)^{2}}{|x-y|} d x d y\right]\right. \\
& \left.\quad+(1-\lambda)\left[\int|\nabla \phi|^{2} d x-\frac{1.68 U N^{1 / 3}}{1-\lambda} \int \phi^{8 / 3} d x\right]\right\} .
\end{aligned}
$$

Again, the first expression in brackets is minimized provided $\phi$ is the minimizer of the Pekar functional with parameter $N(2 \alpha-U) / 2 \lambda$. As for the second term, we first use Sobolev inequality [13], and then find that the minimum of the functional,

$$
3\left(\frac{\pi}{2}\right)^{4 / 3}\left(\int \phi^{6} d x\right)^{1 / 3}-\frac{1.68 U N^{1 / 3}}{1-\lambda} \int \phi^{8 / 3} d x
$$

subject to the restriction $\int \phi^{2} d x=1$ is given by,

$$
-\frac{(0.84)^{2} U^{2} N^{2 / 3}}{3(1-\lambda)^{2}}\left(\frac{2}{\pi}\right)^{4 / 3}
$$

Thus,

$$
\mathcal{P}_{U}^{(N)}(\psi) \geq-N^{3}\left[\frac{c_{p}}{4 \lambda}(2 \alpha-U)^{2}+\frac{(0.84)^{2} U^{2}}{3(1-\lambda) N^{4 / 3}}\left(\frac{2}{\pi}\right)^{4 / 3}\right] .
$$

Putting $\lambda=1-1 / N^{\varepsilon}$, with $0<\varepsilon<4 / 3(N \geq 2)$, it is found that,

$$
\begin{gathered}
-\left[\frac{c_{p}}{4}(U-2 \alpha)^{2} \frac{N^{\varepsilon}}{N^{\varepsilon}-1}+\frac{(0.84)^{2} U^{2}}{3 N^{4 / 3-\varepsilon}}\left(\frac{2}{\pi}\right)^{4 / 3}\right] \leq \frac{\mathcal{E}_{U}^{(N)}(\psi)}{N^{3}} \\
\leq-\frac{c_{p}}{4}\left(U-2 \alpha-\frac{U}{N}\right)^{2},
\end{gathered}
$$

(here the right side is just the upper bound obtained in the equation (26) above) and taking the limit $N \rightarrow \infty$, we conclude the proof of the theorem.

\section{Corollary 2.3.}

i) For all $N$,

$$
-\frac{(1.68)^{2} \alpha^{2}}{3}\left(\frac{2}{\pi}\right)^{4 / 3} N^{5 / 3} \leq \mathcal{E}_{2 \alpha}^{N} \leq-c_{p} \alpha^{2} N
$$


ii) In particular Theorem 2.2 holds at the points 0 and $2 \alpha$.

Proof. The first assertion follows from the continuity of $\mathcal{E}_{U}^{N}$ and the fact that the inequality (32) holds for all $\lambda$ in $(0,1)$. The last one is a consequence of the first and Theorem 2.1.

Remark: The bound (34) is obviously unsatisfactory; it is still open the question about what the correct asymptotic behavior is in this case. However, if the electrons are treated as fermions, the Lieb-Thirring inequality for the kinetic energy [17] may be used, which yields a lower bound proportional to $N$. This has been done by Griesemer and Møller [10], a proof also appears in [4].

\section{ACKNOWLEDGMENT}

This work has been supported by the Iniciativa Científica Milenio, ICM (CHILE) project P07-027-F. The work of RB has also been supported by FONDECYT (Chile) Project 1100679.

\section{REFERENCES}

[1] J. Appel, Polarons. In: Solid State Physics, Advances in Research and Applications, Vol. 21, F. Seitz, D. Turnbull, H. Ehrenreich, ed., Academic Press (1969).

[2] M.D. Donsker, S.R.S Varadhan, Asymptotics for the polaron. Comm. Pure Appl. Math. 36, 505-528 (1983).

[3] R. L. Frank, E. H. Lieb, R. Seiringer, L. Thomas, Bipolaron and N-polaron binding energies, Phys. Rev. Letts. 104, 210402 (2010).

[4] R. L. Frank, E. H. Lieb, R. Seiringer, L. Thomas, Stability and absence of binding for multipolaron systems., Les Publications Mathématiques de l'IHÉS, to appear.

[5] R. L. Frank, E. H. Lieb, R. Seiringer, L. Thomas, Binding, Stability, and Non-binding of Multi-polaron Systems, to appear in the proceedings of the conference QMath11, (Hradec Kralove, Sept. 2010).

[6] H. Fröhlich, Theory of electrical breakdown in ionic crystals. Proc. R. Soc. Lond. A 160, 230-241 (1937).

[7] H. Fröhlich, Electrons in lattice fields. Adv. in Phys. 3, 325-362 (1954).

[8] B. Gerlach, H. Löwen, Analytical properties of polaron systems or: Do polaronic phase transitions exist or not? Rev. Mod. Phys. 63, 63-90 (1991).

[9] M. Griesemer, Exponential decay and ionization thresholds in non-relativistic quantum electrodynamics. J. Funct. Anal. 210, 321-340 (2004).

[10] M. Griesemer, J.S. Møller, Bounds on the minimal energy of translation invariant $N$-polaron systems. Comm. Math. Phys. 297, 283-297 (2010).

[11] M. Hoffmann-Ostenhof, T. Hoffmann-Ostenhof, "Schrödinger inequalities" and asymptotic behavior of the electron density of atoms and molecules. Phys. Rev. A, 16, 1782-1785 (1977).

[12] E.H. Lieb, Existence and uniqueness of the minimizing solution of Choquard's nonlinear equation. Studies in Appl. Math. 57, 93-105 (1977).

[13] E.H. Lieb, M. Loss, Analysis. Graduate Studies in Mathematics, American Mathematical Society (2001).

[14] E.H. Lieb, S. Oxford, An improved lower bound on the indirect Coulomb energy. Int. J. Quant. Chem. 19, 427-439 (1981).

[15] E.H. Lieb, R. Seiringer, The stability of matter in quantum mechanics. Cambridge (2010).

[16] E.H. Lieb, L.E. Thomas, Exact ground state energy of the strong-coupling polaron. Comm. Math. Phys 183, 511-519 (1997). Erratum: ibid. 188, 499-500 (1997).

[17] E.H. Lieb, W. Thirring, Bound for the kinetic energy of fermions which proves the stability of matter. Phys. Rev. Lett. 35, 687-689 (1975). Erratum: ibid. 351116 (1975).

[18] O. Madelung, Introduction to Solid-State Theory. Springer (1981).

[19] T. Miyao, H. Spohn, The bipolaron in the strong coupling limit. Ann. Henri Poincaré 8, 1333-1370 (2007).

[20] S.I. Pekar, Untersuchung über die Elektronentheorie der Kristalle. Berlin: Akademie Verlag (1954).

[21] S.I. Pekar, Research in electron theory of crystals. United States Atomic Energy Commission, Washington, DC (1963). 
[22] S.I. Pekar, O.F. Tomasevich, Theory of F centers. Zh. Eksp. Teor. Fiz. 21, 1218-1222 (1951).

1 Departamento de Física, P. Universidad Católica de Chile, E-mail address: rbenguri@fis.puc.cl

2 Departamento de Matemática, Facultad de Matemáticas, P. Universidad Católica de Chile,

E-mail address: gabley@uc.cl 